OPEN ACCESS

Edited by:

Albert Dahan

Leiden University Medical

Center, Netherlands

Reviewed by:

Giannina Descalzi,

University of Guelph, Canada

Felix Wiedmann,

Heidelberg University

Hospital, Germany

${ }^{*}$ Correspondence:

Logan J. Voss

logan.voss@waikatodhb.health.nz

Specialty section:

This article was submitted to Pharmacological Treatment of Pain,

a section of the journal

Frontiers in Pain Research

Received: 01 December 2021

Accepted: 17 January 2022

Published: 15 February 2022

Citation:

Voss LJ, Harvey MG and Sleigh JW (2022) Non-NMDA Mechanisms of

Analgesia in Ketamine Analogs.

Front. Pain Res. 3:827372.

doi: 10.3389/fpain.2022.827372

\section{Non-NMDA Mechanisms of Analgesia in Ketamine Analogs}

\author{
Logan J. Voss ${ }^{1 *}$, Martyn G. Harvey ${ }^{2}$ and James W. Sleigh ${ }^{1}$ \\ ${ }^{1}$ Anaesthesia Department, Waikato District Health Board, Hamilton, New Zealand, ${ }^{2}$ Emergency Department, Waikato District \\ Health Board, Hamilton, New Zealand
}

Despite 50 years of clinical use and experimental endeavor the anesthetic, analgesic, and psychomimetic effects of ketamine remain to be fully elucidated. While NMDA receptor antagonism has been long held as ketamine's fundamental molecular action, interrogation of bespoke ketamine analogs with known absent NMDA binding, yet profound anesthetic and analgesia fingerprints, suggests alternative targets are responsible for these effects. Herein we describe experimental findings utilizing such analogs as probes to explore ketamine-based analgesic molecular targets. We have focused on two-pore potassium leak channels, identifying TWIK channels as a rational target to pursue further. While the totality of ketamine's mechanistic action is yet to be fully determined, these investigations raise the intriguing prospect of separating out analgesia and anesthetic effects from ketamine's undesirable psychomimesis - and development of more specific analgesic medications.

Keywords: ketamine, analgesia, NMDA - N-methyl-D-aspartate, potassium channels, psychomimetic

\section{INTRODUCTION}

Ketamine is a dissociative anesthetic and potent analgesic. Its well-documented molecular action as a non-competitive NMDA channel antagonist has long been viewed as the likely mediator of its anesthetic and analgesic properties (1). However, this assumption has come under increasing scrutiny with the realization that ketamine affects multiple molecular targets within the central nervous system $(2,3)$. Even so, and despite more than 50 years of clinical use, our understanding of ketamine's molecular level mechanisms of action remains inadequate. This severely stymies "soft" designer drug development targeting ketamine's analgesic and anesthetic profile whilst minimizing its undesirable hallucinogenic properties. The latter is particularly relevant for ketamine's prescription as an analgesic, because effective dosing for analgesia overlaps with its psychotomimetic dose response profile (4).

Our group has been developing and testing bespoke ester analogs of ketamine-with the aim of circumventing ketamine's hallucinogenic side effects by facilitating rapid hydrolysis to inactive carboxylic acid metabolites (5). The hypothesis is that the psychotomimetic window would be shortened due to the rapid degradation of the active compound by tissue esterases. Serendipitously, the family of $30+$ analogs were found to possess a broad range of NMDA receptor binding affinities. When the NMDA affinities are related to their analgesic potencies, it's clear that the analgesic component is not explained by an NMDA blocking action (6) (see Figure 1). One compound in particular, an isopropyl ester ("R5," "SN35563") with similar analgesic potency to ketamine (5) has an NDMA affinity 191 times less than ketamine (8). 


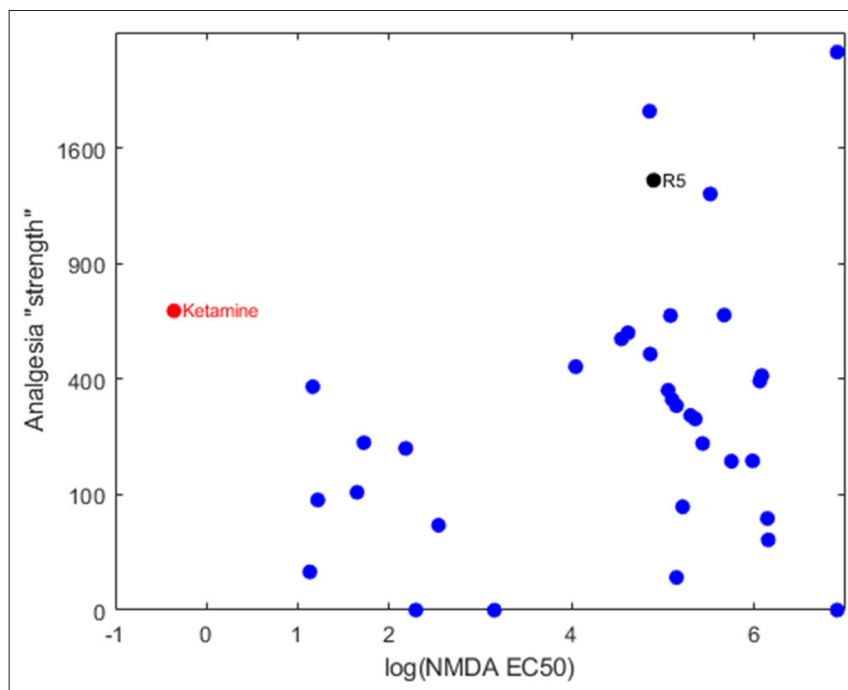

FIGURE 1 | Bi-logarithmic plot of NMDA binding (EC50) vs. analgesia for a series of ketamine analogs. The red dot is the parent ketamine, the black dot is R5 and the blue dots are other ketamine-ester variants. There is no correlation, suggesting that NMDA has a negligible role in production of analgesia for these compounds. Determination of NMDA inhibition was carried out by commercial provider (Eurofins Panlabs Taiwan, Ltd. Pharmacology Laboratories) according to reported procedure (7). MK-801 $\{[5 R, 10 S]-[+]$ -5-methyl-10,11-dihydro-5H-dibenzo [a,d]cyclohepten-5,10-imine\}, Ketamine and ketamine analogs were tested for competitive binding against the radioligand $5 \mathrm{nM}\left[{ }^{3} \mathrm{H}\right]-\mathrm{MK}-801$ in wistar rat brain preparations, incubated in $5 \mathrm{mM}$ Tris- $\mathrm{HCL}$ at $\mathrm{pH} 7.4$ for $3 \mathrm{~h}$ at $25^{\circ} \mathrm{C}$. Radioligand displacement was used to determine ligand binding affinity to calculate $\mathrm{IC}_{50}$.

This confirms beyond any doubt that ketamine-based analgesia can occur independent of its NMDA antagonism. This raises the tantalizing prospect of investigating analgesia mechanisms in a ketamine model void of NMDA activity-and development of more specific analgesic medications if drug targets are known.

These discoveries opened the door to using R5 as an experimental tool for investigating possible molecular targets for ketamine analgesia, without the confounding influence of NMDA antagonism. With an almost endless number of potential targets, we have focused primarily on two-pore potassium "leak" (K2P) channels. Potassium channels are ubiquitous in all living organisms and in humans comprise at least 80 different subtypes. K2P channels, in particular, form a 15member subclass that provide background neuronal currents responsible for stabilizing the resting membrane potential (9). Our attention was drawn to K2P channels for two reasons. Firstly, K2P channels are emerging as important potential targets for nociceptive regulation (10). Secondly, and more importantly, K2P channels have been previously implicated in ketamine's mechanism of action (11). In the sections that follow we will outline a series of ex vivo and in vitro studies exploring K2P channels as a possible ketamine analog (R5) target.

\section{EX VIVO BRAIN SLICE EFFECTS OF R5}

\section{Cerebral Cortex}

We first established that R5 behaved similarly to ketamine in ex vivo mouse cortical slices. Ketamine strongly inhibits cortical seizure-like event (SLE) activity, an effect that is barium chloride $\left(\mathrm{BaCl}_{2}\right)$ and urethane sensitive-implying involvement of $\mathrm{K} 2 \mathrm{P}$ channels (11).

The methodology for slice preparation, electrophysiological recording and pharmacology manipulation is detailed elsewhere (11). Briefly, $400 \mu \mathrm{m}$ coronal mouse brain slices were prepared from adult male and female C57 mice. The tissue slices were prepared in HEPES-buffered "normal" artificial cerebrospinal fluid (aCSF) (oxygenated with 95\% oxygen), before being transferred to a submersion-style perfusion bath replenished with oxygenated aCSF void of magnesium ions ("no-Mg aCSF") by gravity-feed at a rate of $5 \mathrm{~mL} / \mathrm{min}$. The lack of magnesium ions in solution activates the tissue by unblocking NMDA receptors, resulting in the generation of repeating population bursts known as seizure-like events (SLEs). Spontaneous extracellular field potentials were recorded from layer IV of the somatosensory cortex, amplified and filtered (low pass $300 \mathrm{~Hz}$ and high pass $1 \mathrm{~Hz}$ ) and stored for later analysis after analog-digital conversion. All test drugs were added directly to pre-oxygenated no$\mathrm{Mg}$ aCSF to the required concentrations. The main outcome variable was the change in SLE inter-event frequency, which is reliably reduced by ketamine and other anesthetics (11, 12). Because of degradation of $\mathrm{R} 5$ by tissue esterases, a much higher R5 concentration $(100 \mu \mathrm{g} / \mathrm{mL})$ than ketamine $(4 \mu \mathrm{g} / \mathrm{mL})$ was required to achieve a similar reduction in SLE frequency. The pan carboxyl-esterase inhibitor 2,2'-Thenil (13) confirmed that esterase activity was largely responsible for this relative lack of potency, restoring R5 effectiveness in this model at $15 \mu \mathrm{g} / \mathrm{mL}$, while having no effect on ketamine action at $4 \mu \mathrm{g} / \mathrm{mL}$.

Potassium channel effects of $\mathrm{R} 5$ were probed using $\mathrm{BaCl}_{2}$ and urethane. Urethane is a central nervous system depressant (anesthetic) that activates barium-sensitive potassium leak channels (14). $\mathrm{BaCl}_{2}$ at low concentrations $(<200 \mu \mathrm{M})$ blocks potassium leak currents, with TREK and TWIK-mediated currents particularly implicated (14-16). Effects were similar to those reported for ketamine, where SLE frequency reduction was blocked by barium chloride and enhanced by urethane (11). $\mathrm{BaCl}_{2}(200 \mu \mathrm{M})$ eliminated the $\mathrm{R} 5$ suppression of SLE frequency. At a concentration sufficient to significantly reduce cortical neuronal spike rates in vitro [>10 mM (14)], we found that urethane $(20 \mathrm{mM})$ reduced SLE frequency by $27(13) \%$, but the reduction was significantly increased to 60 (17)\% when combined with $\mathrm{R} 5$. In keeping with a potassium leak channel enhancing effect, reducing the potassium concentration in the aCSF enhanced the effect of R5. The data implicate opening of barium-sensitive TREK and/or TWIK K2P channels in the cortical depressant effect of R5 in cortical slices. This was examined further with the TREK-1 blocker, spadin (18). At two concentrations (7 and $25 \mu \mathrm{M})$, spadin had no effect in isolation, nor did it modify the effect of R5 on 
SLE frequency-seemingly eliminating cerebrocortical TREK-1 channels as a target.

\section{Differential Sensitivity, Basolateral Amygdala vs. Cerebral Cortex}

Subanaesthetic concentrations of ketamine have been reported to have both cortical and subcortical effects (19). In an earlier transcriptome study we identified two central subcortical nuclei that were particularly sensitive to R5 for large-scale gene expression changes, the basolateral amygdala (BLA) and paraventricular nucleus of the thalamus (PVT) (20). This suggested that R5 was likely to be acting centrally with differential sensitivity at specific subcortical nuclei. To test this in mouse brain slices, cortical slices were prepared as above and selected for those containing the BLA. No-Mg SLE activity was recorded concurrently from the auditory cortex and the BLA by overlaying a $2 \times 16$ rectangular electrode array $(50 \mu \mathrm{m}$ stainless steel, $500 \mu \mathrm{m}$ row separation, and $250 \mu \mathrm{m}$ electrode separation). Extracellular field potentials were recorded using a Blackrock Cereplex system (high pass filter $0.3 \mathrm{~Hz}$ and low pass filter $250 \mathrm{~Hz}$ ) with $1 \mathrm{kHz}$ sampling.

After baseline recording to establish stable SLE activity, R5 was perfused $(5 \mathrm{ml} / \mathrm{min})$ for 20 mins at a time at sequential increasing concentrations of 20 and $30 \mu \mathrm{g} / \mathrm{mL}$ and for $10 \mathrm{~min}$ at $40 \mu \mathrm{g} / \mathrm{mL}$. Because SLE parameters (frequency, length, and amplitude) appeared to be affected differentially, a composite measure equating to SLE frequency * length * amplitude was quantified for each recording to give a measure of overall activity (normalized to percent change from baseline).

SLE activity was synchronous across the cerebral cortex and the BLA, consistent with findings showing that the auditory cortex interacts directly with the BLA in fear learning (21). The clear finding was that BLA activity was significantly more sensitive to R5 than cortical activity (Figure 2). In fact, no significant change was identified in the cortex, while a clear reduction was noted in the BLA from the $30 \mu \mathrm{g} / \mathrm{mL}$ time point. The BLA effect reversed by the end of a $40 \mathrm{~min}$ wash period.

Together, the brain slice results suggest that R5 acts centrally and with differential sensitivity at cortical and subcortical locations. The BLA, which is involved in fear conditioning to aversive stimuli (22) may be specifically implicated. In vivo "adhesive removal test" (23) experiments strengthen the case for involvement of the limbic system in R5 analgesia, showing that rats administered subanaesthetic doses of R5 were behaviourally normal, but oblivious to what would ordinarily be the noxious stimulus of having a small piece of sticky tape adhered to a forepaw.

The BLA was chosen over the PVT for electrophysiological study because of its suitability for investigation using our established low-magnesium cortical slice model. That is, the BLA is interconnected with the auditory cerebral cortex (21) and generates low-magnesium seizure-like event activity that is synchronized (connected) with activity recorded from the auditory cortex. This allowed for concurrent recording from both regions and direct pairwise comparison of the R5 effect. We would emphasize that this does not preclude the PVT, or potentially other subcortical nuclei, from important mechanistic roles in the action of R5. It should also be noted that the cortical slice model has been principally validated against the hypnotic effects of anesthetic drugs-including the family of ketamine analogs (24). The correlation between ketamine analogs and in vivo hypnotic potency and SLE slice effect is robust $\left(R^{2}=36 \%\right.$, $p=0.003)$, but is much weaker for the analgesic effect $\left(R^{2}=\right.$ $17 \%, p=0.06$ ). The bearing of these findings on mechanisms of ketamine analgesia must therefore be substantiated in a more relevant model. The section that follows describes in vivo experiments designed to confirm a central K2P mechanism in a validated analgesia model.

\section{INTRAVENTRICULAR MICROINJECTION OF R5 AND EFFECT OF BARIUM CHLORIDE}

Drug microinjection is a technique for the direct mechanical delivery of small drug volumes to specific stereotaxically identified brain regions (25). Because the BLA (and PVT) appear to be highly sensitive to R5, we took advantage of both nuclei having paraventricular aspects (the lateral ventricles and third ventricle for BLA and PVT, respectively) and quantified the effect of intraventricular R5 microinjection on pain responses in rats. Tests were repeated with barium chloride pretreatment. Based on the R5 brain slice findings, we hypothesized that $\mathrm{BaCl}_{2}$ would antagonize a central analgesic effect of R5. Rats were used for this investigation because previous behavioral investigations had utilized the rat model $(5,6,8,26)$. The reason for this is the necessity for secure intravenous tail vein access for drug delivery, which limits drug metabolism by tissue esterases (27). Tail vein drug delivery is not practicable in the smaller mouse model.

Chronically instrumented adult female Sprague Dawley rats were studied. For direct brain cannula implantation rats were anesthetized with isoflurane $2 \%$ then stereotaxically implanted unilaterally (right) with one 26-gauge cannula into the anterior horn of the lateral ventricle. Cannulas were held in place with dental cement, and patency maintained with occlusive stylets. A tail flick analgesia meter (Colombus Instruments, Colombus, Ohio) was used to determine pain sensitivity. Radiant heat was applied using a shutter-controlled lamp as a heat source focused on a spot located $6-8 \mathrm{~cm}$ from the tip of the tail. The intensity of the beam was set at a level producing basal latency times between 3 and $4.5 \mathrm{~s}$. To prevent thermal tissue injury the cut off time was set at $10 \mathrm{~s}$. A digital response time indicator with a resolution of $0.1 \mathrm{~s}$ measured the time from initiation of stimulus until tail withdrawal (the flick). Tail flick latency (TFL) was calculated as a percentage of the maximum possible effect (MPE) such that:

$$
\begin{aligned}
\% \mathrm{MPE}= & {[\mathrm{TFL}(\text { post }- \text { drug })-\mathrm{TFL}(\text { pre }- \text { drug }) /} \\
& 10 \mathrm{~s}-\mathrm{TFL}(\text { pre }- \text { drug })] \times 100 \%
\end{aligned}
$$




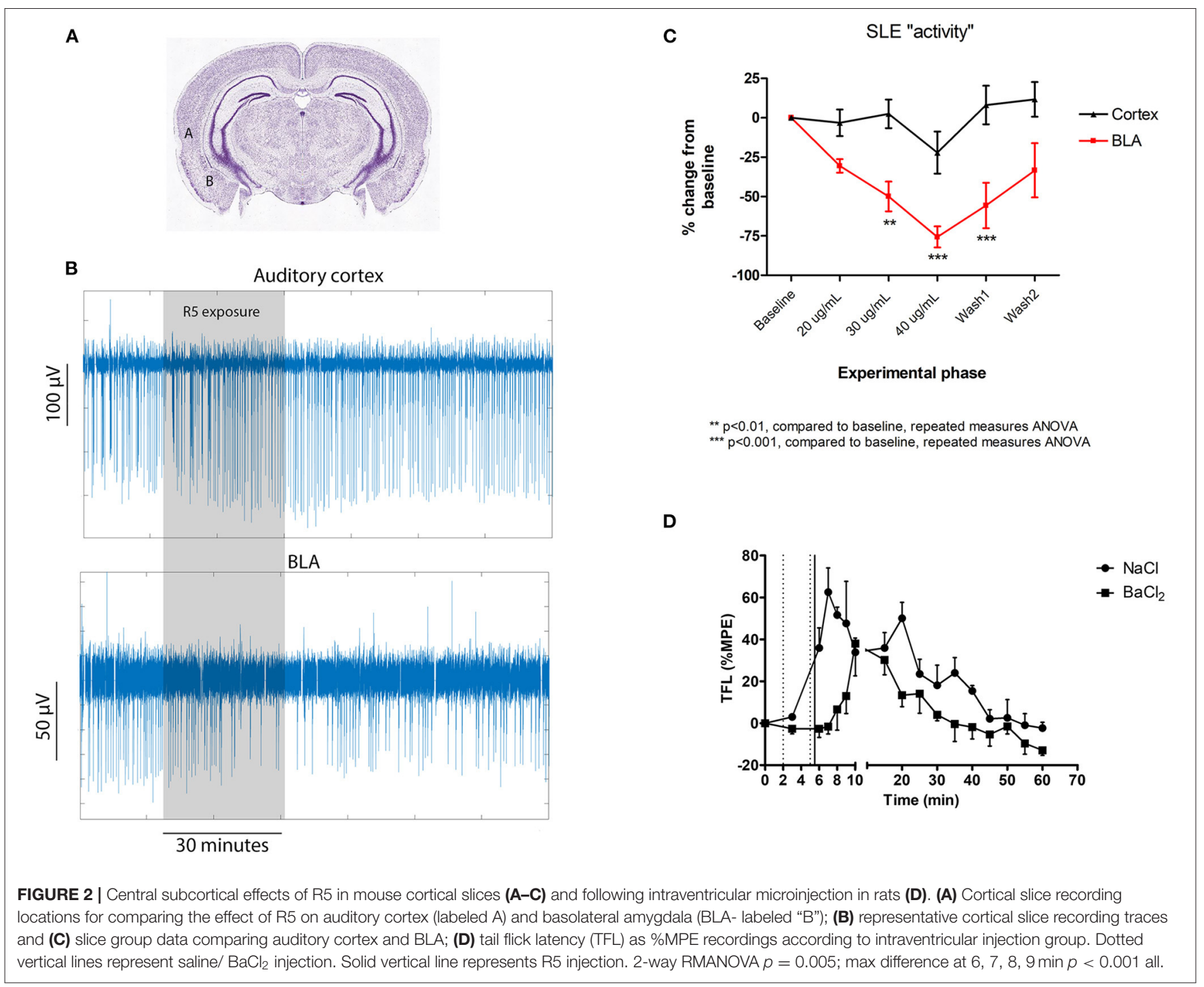

For intraventricular injections, solutions were loaded into $30 \mathrm{~cm}$ lengths of PE-50 tubing attached at one end to a $10 \mu \mathrm{L}$ Hamilton syringe prefilled with test agents. All injections were delivered by $1.0 \mu \mathrm{L}$ bolus over $30 \mathrm{~s}$. Following assessment of baseline tail flick latency (time 0) rats underwent intraventricular injection of $1 \mu \mathrm{L}$ saline $(0.9 \%)$ or $1 \mu \mathrm{L} \mathrm{BaCl} 2(10 \mathrm{mg} / \mathrm{mL})$ at time equals $2 \mathrm{~min}$. TFL was reassessed at $3 \mathrm{~min}$. Further 1 $\mu \mathrm{L}$ injections of saline or barium solutions were undertaken at $5 \mathrm{~min}$ and followed immediately by intraventricular injection of $1 \mu \mathrm{L}$ R5 (10 mg/mL). TFL evaluation was undertaken at $1 \mathrm{~min}$ intervals from time 6 to $10 \mathrm{~min}$, then at $5 \mathrm{~min}$ intervals thereafter to $60 \mathrm{~min}$.

TFL responses as \%MPE are presented in Figure 2D. Intraventricular microinjection of R5 had a clear analgesic effect, seen as a significant increase in tail flick latency lasting for nearly 60 min. Importantly, pretreatment with $\mathrm{BaCl}_{2}$ antagonized the early phase of R5 analgesia, delaying the increase in TFL by 8 $10 \mathrm{~min}$. The data confirms the cortical slice results showing that
R5 analgesia is centrally mediated and barium-sensitive. While both the BLA and PVT have paraventricular regions, many other nuclei could have been affected by intraventricular R5 injection. These results do not exclude involvement of other regions such as the periaqueductal gray and pretectal areas. A more targeted suite of experiments will be necessary to confirm involvement of specific nuclei.

\section{ELECTROPHYSIOLOGICAL QUANTIFICATION OF TREK-1 AND TREK-2 EFFECTS OF R5}

The cortical slice work suggested that TREK-1 K2P receptors were not relevant to the cortical depressant effect of R5. To unequivocally rule out TREK channels as an R5 target, recombinant TREK-1 ( $\left.\mathrm{K}_{2 \mathrm{P}} 2.1\right)$ and TREK-2 ( $\left.\mathrm{K}_{2 \mathrm{P}} 10.1\right)$ expressing cells (host cell mouse HEK) were electrophysiologically 
tested using the voltage clamp technique in patch clamped whole cells.

Outward potassium currents were measured at voltage steps from -80 to $+100 \mathrm{mV}(300 \mathrm{~ms})$. The testing protocol consisted of first adding extracellular physiological solution (control period) twice $(2-3 \mathrm{~min})$, followed by one concentration of test compound $(2-3 \mathrm{~min})$ and a saturating inhibitory concentration of specific channel blocker $\mathrm{BaCl}_{2}(10 \mathrm{mM})$. For all test compounds, nine concentrations were tested, $0.1,0.3,1,3,10,30,100,300,1,000 \mu \mathrm{M}$. Data were normalized using the last point obtained during the precompound saline application as the zero value (0) and maximum block by the reference blocker $\left(\mathrm{BaCl}_{2}\right)$ as the top value (1.0).

All cells used in these experiments showed functional TREK-1 and TREK-2 channels which were blocked by the addition of a high concentration of Tetrahexylammonium (THA) and Quinidine, respectively, validating the assay. R5 induced a weak concentration dependent block of TREK1 and TREK-2 channels, with an $\mathrm{IC}_{50}$ of $24 \mu \mathrm{g} / \mathrm{mL}$ for TREK-1 and $104 \mu \mathrm{g} / \mathrm{mL}$ for TREK-2. Ketamine was also tested and had no effect on TREK-2 and a very weakly blocked TREK-1 (EC $50118 \mu \mathrm{g} / \mathrm{mL})$. There are two telling observations to make from these results. Firstly, the $\mathrm{EC}_{50}$ values for the TREK channel effects were up to 2 orders of magnitude more than the therapeutic concentrations for these agents. Secondly, the effect noted was channel blockadewhereas channel opening was hypothesized to explain the R5 depressant effect. Based on these results, TREK K2P channels can be ruled out as a therapeutic target for R5 and ketamine.

\section{OTHER POTENTIAL TARGETS}

As we've discussed, barium sensitivity at low concentrations $(<200 \mu \mathrm{M})$ implies involvement of TREK or TWIK-mediated currents (14-16). With TREK channels effectively ruled out, TWIK channels remain the most likely K2P target for R5. Cesium chloride $(\mathrm{CsCl})$ is ineffective at TWIK-1 channels in the low $\mathrm{mM}$ range (28), while barium $\left(\mathrm{Ba}^{+2}\right)$ strongly blocks TWIK-1 currents in the $\mu \mathrm{M}$ range (28). Thus, $\mathrm{Cs}^{+}$ and $\mathrm{Ba}^{+2}$ can be used to test for TWIK-1 specificity. In our cortical slice experiments, $2 \mathrm{mM} \mathrm{CsCl}$ was ineffectual on R5, strengthening the case for TWIK channel involvement. Experimental electrophysiological verification of TWIK channel involvement in $\mathrm{R} 5$ and ketamine action remains to be undertaken.

While our focus in the presented studies has been K2P channels, numerous other potential analgesia targets could be relevant. We touched on a subset of these using commercial channel screens but failed to identify any R5 molecular targets at clinically relevant concentrations. These include radioligand binding studies on calcium L-type channels, $\mathrm{GABA}_{\mathrm{A}}$ receptors and $\delta_{1}$ and $\mu$ opiate receptors-and patch clamp functional assay of HCN1 potassium channels. Binding profiles were sourced from Eurofins Panlabs Ltd.
(Taipei, Taiwan). Duplicate studies were undertaken at concentrations $10 \mu \mathrm{M}, 1 \mu \mathrm{M}, 0.1 \mu \mathrm{M}$, and $10 \mathrm{nM}$, for the compounds. Detailed explanations of the methodology employed in potential-target profiling can be found at http://www.eurofinspanlabs.com/Panlabs using the number listed in parentheses below: calcium channel L-type, opiate $\delta 1$ (260130); opiate $\mu$ (260410); norepinephrine uptake (302000); and hyperpolarization-activated cyclic nucleotide-gated ion channel, HCN1 (952727). Greater than 50\% binding inhibition was deemed significant. Reference standards were run as an integral part of each assay to ensure the validity of the results obtained.

\section{DISCUSSION}

The role of the NMDA receptors in ketamine analgesia has been largely unquestioned. This view has been strengthened by the likes of compounds such as phencyclidine and dizolcipine, which potently bind to the NMDA receptor complex and are strong analgesics (29). Of the ketamine enantiomers, $\mathrm{S}(+)$-ketamine has about 3-fold more analgesic potency than R(-)-ketamine, which has been attributed to its increased NMDA affinity (30). Our data indicates that targets other than the NMDA receptor are important mediators of analgesia for ketamine analogs. It's likely that NMDA binding is only one component of a ketamine-related analgesic cascade, and that ketamine analog compounds have varying degrees of additional actions that contribute to the overall analgesic activity.

Based on relative sensitivity to barium, caesium, and urethane, the TWIK-1 channel is a plausible component of a ketamine-analog analgesic mechanism. TWIK-1 was the first mammalian K2P channel identified (31), so-called because it is a weakly inward rectifying two-pore potassium channel. It is widely expressed in the brain where, like other K2P channels, it is thought to provide a leak potassium conductance that stabilizes the membrane potential (17). Further work demonstrating positive allosteric modulation of the TWIK-1 channel by ketamine is required to confirm our findings. These are challenging experiments however, because heterologous expression of TWIK-1 proteins in Xenopus oocytes or cultured mammalian cells results in low channel functionality (32-34). This impediment to studying the functional characteristics of TWIK-1 may be one of the reasons this channel has not been favored to date as an anesthetic target (35). Our studies support a centrally located analgesic mechanism, the primary brain regions most likely linked to the limbic system-principally the basolateral amygdala and cerebral cortex. The implication here is that the analgesic potential of ketamine analogs may reside in their capacity to moderate the offensiveness of pain signals entering the brain, more than inhibit their propagation. This is contrary to the view that ketamine acts at least in part as a local anesthetic via NMDA blockade (36).

In summary, challenging the widely view that ketamine analgesia is due to NMDA blockade is the first step toward separating out analgesia from its undesirable psychomimetic side effects. Identification of analgesic molecular targets will aid the 
development of more potent and specific analgesic medications. We have used ketamine analogs that are void of both NMDA action and psychomimetic effects as model compounds to begin differentiating these effects. In particular, centrally expressed K2P channels should be carefully scrutinized as potential targets for ketamine analgesia.

\section{DATA AVAILABILITY STATEMENT}

The raw data supporting the conclusions of this article will be made available by the authors, without undue reservation.

\section{REFERENCES}

1. Orser BA, Pennefather PS, MacDonald JF. Multiple mechanisms of ketamine blockade of N-methyl-D-aspartate receptors. Anesthesiology. (1997) 86:90317. doi: 10.1097/00000542-199704000-00021

2. Li L, Vlisides PE. Ketamine: 50 years of modulating the mind. Front Hum Neurosci. (2016) 10:612. doi: 10.3389/fnhum.2016.00612

3. Sleigh J, Harvey M, Voss L, Denny B. Ketamine - more mechanisms of action than just NMDA blockade. Trends Anaesth Crit Care. (2014) 4:2. doi: 10.1016/j.tacc.2014.03.002

4. Bowdle TA, Radant AD, Cowley DS, Kharasch ED, Strassman RJ, Roy-Byrne PP. Psychedelic effects of ketamine in healthy volunteers: relationship to steady-state plasma concentrations. Anesthesiology. (1998) 88:82-8. doi: 10.1097/00000542-199801000-00015

5. Jose J, Gamage SA, Harvey MG, Voss LJ, Sleigh JW, Denny WA. Structureactivity relationships for ketamine esters as short-acting anaesthetics. Bioorganic Med Chem. (2013) 21:5098-106. doi: 10.1016/j.bmc.2013. 06.047

6. Dimitrov IV, Harvey MG, Voss LJ, Sleigh JW, Bickerdike MJ, Denny WA. Structure-activity relationships for the anaesthetic and analgaesic properties of aromatic ring-substituted ketamine esters. Molecules. (2020) 25:122950. doi: 10.3390/molecules 251 22950

7. Reynolds IJ, Murphy SN, Miller RJ. 3H-labeled MK-801 binding to the excitatory amino acid receptor complex from rat brain is enhanced by glycine. Proc Natl Acad Sci USA. (1987) 84:7744-8. doi: 10.1073/pnas.84.2 1.7744

8. Dimitrov IV, Harvey MG, Voss LJ, Sleigh JW, Bickerdike MJ, Denny WA. Ketamine esters and amides as short-acting anaesthetics: structure-activity relationships for the side-chain. Bioorganic Med Chem. (2019) 27:122631. doi: 10.1016/j.bmc.2019.02.010

9. Goldstein SAN, Bayliss DA, Kim D, Lesage F, Plant LD, Rajan S. LV nomenclature and molecular relationships of two-P potassium channels. Pharmacol Rev. (2005) 57:527-40. doi: 10.1124/pr.57.4.12

10. Gada K, Plant LD. Two-pore domain potassium channels: emerging targets for novel analgesic drugs: IUPHAR Review 26. Br J Pharmacol. (2019) 176:256-66. doi: 10.1111/bph.14518

11. Voss LJ, Karalus S, Englund V, Sleigh JW. Ketamine action in the in vitro cortical slice is mitigated by potassium channel blockade. Anesthesiology. (2018) 128:1167-74. doi: 10.1097/ALN.0000000000002147

12. Voss LJ, Sleigh JW. A metabolic mechanism for anaesthetic suppression of cortical synaptic function in mouse brain slices-a pilot investigation. Int $J$ Mol Sci. (2020) 21:1-13. doi: 10.3390/ijms21134703

13. Hyatt JL, Stacy V, Wadkins RM, Yoon KJP, Wierdl M, Edwards CC, et al. Inhibition of carboxylesterases by benzil (diphenylethane-1,2-dione) and heterocyclic analogues is dependent upon the aromaticity of the ring and the flexibility of the dione moiety. J Med Chem. (2005) 48:554350. doi: $10.1021 / \mathrm{jm} 0504196$

14. Sceniak MP, MacIver MB. Cellular actions of urethane on rat visual cortical neurons in vitro. J Neurophysiol. (2006) 95:3865-74. doi: 10.1152/jn.01196.2005

\section{ETHICS STATEMENT}

The animal study was reviewed and approved by University of Waikato Animal Ethics Committee.

\section{AUTHOR CONTRIBUTIONS}

LV wrote the manuscript and conducted the cortical slice experiments. $\mathrm{MH}$ conducted the in vivo experiments and wrote the manuscript. JS wrote the manuscript. All authors contributed to the article and approved the submitted version.

15. Sanders RD, Patel N, Hossain M, Ma D, Maze M. Isoflurane exerts antinociceptive and hypnotic properties at all ages in Fischer rats. Br J Anaesth. (2005) 95:393-9. doi: 10.1093/bja/aei182

16. Patel AJ, Honoré E. Properties and modulation of mammalian $2 \mathrm{P}$ domain $\mathrm{K}+$ channels. Trends Neurosci. (2001) 24:33946. doi: 10.1016/S0166-2236(00)01810-5

17. Lesage F, Lazdunski M. Molecular and functional properties of twopore-domain potassium channels. Am J Physiol Ren Physiol. (2000) 279. doi: 10.1152/ajprenal.2000.279.5.F793

18. Borsotto M, Veyssiere J, Moha Ou Maati H, Devader C, Mazella J, Heurteaux C. Targeting two-pore domain $\mathrm{K}+$ channels TREK-1 and TASK-3 for the treatment of depression: a new therapeutic concept. Br J Pharmacol. (2015) 172:771-84. doi: 10.1111/bph.12953

19. Rivolta D, Heidegger T, Scheller B, Sauer A, Schaum M, Birkner $\mathrm{K}$, et al. Ketamine dysregulates the amplitude and connectivity of high-frequency oscillations in cortical-subcortical networks in humans: evidence from resting-state magnetoencephalographyrecordings. Schizophr Bull. (2015) 41:1105-14. doi: 10.1093/schbul/ sbv051

20. Jacobson GM, Voss LJ, Klockars A, Bird S, Dimitrov I, Denny WA, et al. Transcriptional changes in response to ketamine ester-analogs SN 35210 and SN 35563 in the rat brain. BMC Genom. (2019) 20:6. doi: 10.1186/s12864-019-5649-6

21. Cambiaghi M, Renna A, Milano L, Sacchetti B. Reversible inactivation of the higher order auditory cortex during fear memory consolidation prevents memory-related activity in the basolateral amygdala during remote memory retrieval. Front Behav Neurosci. (2017) 11:1-11. doi: 10.3389/fnbeh.2017.00138

22. Krabbe S, Gründemann J, Lüthi A. Amygdala inhibitory circuits regulate associative fear conditioning. Biol Psychiatry. (2018) 83:800-9. doi: 10.1016/j.biopsych.2017.10.006

23. Bouet V, Boulouard M, Toutain J, Divoux D, Bernaudin M, Schumann-Bard $\mathrm{P}$, et al. The adhesive removal test: a sensitive method to assess sensorimotor deficits in mice. Nat Protoc. (2009) 4:1560-4. doi: 10.1038/nprot.2009.125

24. Voss LJ, Harvey MG, Sleigh JW. Inhibition of astrocyte metabolism is not the primary mechanism for anaesthetic hypnosis. Springerplus. (2016) 5:2734. doi: 10.1186/s40064-016-2734-z

25. Peterson SL. Drug microinjection in discrete brain regions. Kopf Carr. (1998) 50:2-7.

26. Harvey M, Sleigh J, Voss L, Bickerdike M, Dimitrov I, Denny W. KEA1010, a ketamine ester analogue, retains analgesic and sedative potency but is devoid of psychomimetic effects. BMC Pharmacol Toxicol. (2019) 20:85. doi: 10.1186/s40360-019-0374-y

27. Harvey M, Sleigh J, Voss L, Jose J, Gamage S, Pruijn F, et al. Development of rapidly metabolized and ultra-short-acting ketamine analogs. Anesth Analg. (2015) 121:925-33. doi: 10.1213/ANE.00000000000 00719

28. Lesage F, Lauritzen I, Duprat F, Reyes R, Fink M, Heurteaux C, et al. The structure, function and distribution of the mouse TWIK-1 K+ channel. FEBS Lett. (1997) 402:28-32. doi: 10.1016/S0014-5793(96)0 1491-3 
29. Coderre TJ. Potent analgesia induced in rats by combined action at PCP and polyamine recognition sites of the NMDA receptor complex. Eur J Neurosci. (1993) 5:390-3. doi: 10.1111/j.1460-9568.1993.tb00506.x

30. Sinner B, Graf BM. Ketamine. Handb Exp Pharmacol. (2008) 31333. doi: 10.1007/978-3-540-74806-9_15

31. Lesage F, Guillemare E, Fink M, Duprat F, Lazdunski M, Romey G, et al. TWIK-1, a ubiquitous human weakly inward rectifying $\mathrm{K}+$ channel with a novel structure. EMBO J. (1996) 15:1004-11. doi: 10.1002/j.1460-2075.1996.tb00437.x

32. Chatelain FC, Bichet D, Douguet D, Feliciangeli S, Bendahhou S, Reichold M, et al. TWIK1, a unique background channel with variable ion selectivity. Proc Natl Acad Sci USA. (2012) 109:5499-504. doi: 10.1073/pnas.1201132109

33. Enyedi P, Czirjak G. Molecular background of leak K+ currents: two-pore domain potassium channels. Physiol Rev. (2010) 90:559-605. doi: 10.1152/physrev.00029.2009

34. O'Kelly I. Endocytosis as a mode to regulate functional expression of twopore domain potassium (K2P) channels. Pflugers Arch Eur J Physiol. (2015) 467:1133-42. doi: 10.1007/s00424-014-1641-9

35. Steinberg EA, Wafford KA, Brickley SG, Franks NP, Wisden W. The role of K2P channels in anaesthesia and sleep. Pflugers Arch Eur J Physiol. (2014) 467:907-16. doi: 10.1007/s00424-014-1654-4
36. Pai A, Heining M. Ketamine. Contin Educ Anaesthesia Crit Care Pain. (2007) 7:59-63. doi: 10.1093/bjaceaccp/mkm008

Conflict of Interest: The authors declare that the research was conducted in the absence of any commercial or financial relationships that could be construed as a potential conflict of interest.

Publisher's Note: All claims expressed in this article are solely those of the authors and do not necessarily represent those of their affiliated organizations, or those of the publisher, the editors and the reviewers. Any product that may be evaluated in this article, or claim that may be made by its manufacturer, is not guaranteed or endorsed by the publisher.

Copyright (c) 2022 Voss, Harvey and Sleigh. This is an open-access article distributed under the terms of the Creative Commons Attribution License (CC BY). The use, distribution or reproduction in other forums is permitted, provided the original author(s) and the copyright owner(s) are credited and that the original publication in this journal is cited, in accordance with accepted academic practice. No use, distribution or reproduction is permitted which does not comply with these terms. 